\title{
Servo design of a vertical axis drive using dual linear motors for high speed electric discharge machining
}

\author{
Min-Fu Hsieh ${ }^{\mathrm{a}, *}$, Chin-Juei Tung ${ }^{\mathrm{b}}$, Wu-Sung $\mathrm{Yao}^{\mathrm{c}}$, Meng-Chieh $\mathrm{Wu}^{\mathrm{a}}$, Yunn-Shiuan Liao ${ }^{\mathrm{b}}$ \\ ${ }^{a}$ Department of Systems and Naval Mechatronic Engineering, National Cheng Kung University, No. 1, Ta-Hsueh Road, Tainan 70101, Taiwan, ROC \\ ${ }^{\mathrm{b}}$ Department of Mechanical Engineering, National Taiwan University, Taipei 10617, Taiwan, ROC \\ ${ }^{\mathrm{c}}$ Department of Mechanical Engineering, National Cheng Kung University, Tainan 70101, Taiwan, ROC
}

Received 2 March 2006; received in revised form 16 May 2006; accepted 28 May 2006

Available online 21 July 2006

\begin{abstract}
This paper proposes a synchronous control scheme for a linear servo system applied to the vertical axis drive of a die-sinking electric discharge machine (EDM) tool. The investigated vertical axis drive is constructed with dual parallel linear motors, which are arranged to jointly drive the feed axis for improvement of the overall thrust and structural stiffness. A pneumatic cylinder is employed to compensate the gravitational effect of the feed axis with its electrode and holder. A mechanical coupling is designed to firmly bridge the two linear motors and carry the feed axis. Therefore, synchronous control for the motors is critical for not only position accuracy, but also machine safety. Moreover, by controlling the thrust outputs and positions of the motors to be as equal as possible, the potential "pull and drag" effect between the motors can be reduced and loads can also be equally shared. The proposed "position/thrust hybrid synchronous control" scheme is applied to the EDM to achieve high-speed, accurate machining, and the experimental results show that the synchronization error between the two parallel motors and the positioning accuracy are both satisfactory when operated under highspeed conditions.
\end{abstract}

(C) 2006 Elsevier Ltd. All rights reserved.

Keywords: Linear motor; Synchronous control; Electric discharge machine; Thrust control

\section{Introduction}

Linear motors, with their non-contact energy translation and high acceleration, have been increasingly applied to various areas requiring high-accuracy and high-speed motions. For application to machine tools, linear motors are often arranged with the so-called "box-in-box" layout to increase the thrust and stiffness of the system, and hence the level of synchronization is crucial for any pair of parallel motors to drive a feed axis [1,2]. This implies that the control problem for such a configuration lies not only in the achievement of satisfactory positioning accuracy, but also in the elimination of the synchronization error between the two parallel motors. In addition to the required performances, safety is another major concern

\footnotetext{
${ }^{*}$ Corresponding author. Tel.: + $88662757575 \times 63537$; fax: +88662747019 .

E-mail address: mfhsieh@mail.ncku.edu.tw (M.-F. Hsieh).
}

as damage can occur when significant synchronization error between the two motors takes place. Therefore, appropriate synchronous control techniques are demanded to reduce such errors, achieve satisfying accuracy and reduce potential hazards.

For related research on synchronous control, Lorenz and Schmidt [3] classify the control techniques into three categories, and they are: (a) synchronized master command generator control, (b) conventional master-slave motion control, and (c) relative dynamic stiffness motion control. For category (a), the two motors with individual control loops are fed with the same commands. However, the synchronization of the two motors cannot be guaranteed, and thus this technique may not be suitable for the case with mechanical coupling between the motors. For the master-slave control technique in category (b), the command of the slave motor is given from the master motor output so that the slave follows the master with an inevitable error unless appropriate compensation is made. 
For category (c), a compensator is employed based on the position/velocity differences between the master and slave motors to achieve synchronization. A technique named the "tandem control" [4] proposed by FANUC Ltd. applies two rotary motors to jointly drive a single axis of motion. SIEMENS [5] also develops a master-slave control technique for rotary motors based on speed/torque coupling. However, the torque allotment between the mechanically coupled motors is not discussed. Yang et al. [6] present a new identification technique for synchronous control of two linear motors with mechanical coupling. Each of the above-mentioned techniques possesses its own features and applications.

For applications of linear motors to electrical discharge machining, Cetin et al. [7] experimentally investigate the effects of electrode jump parameters on machining speed and depth in linear-motor-equipped electrical discharge machining. Zhao et al. [8] develop a geometric model for the die-sinking process equipped with linear motors. However, none of the above papers discuss the control issue for linear motors applied to this process.

Therefore, this paper proposes a control technique, named the "position/thrust hybrid synchronous control" for a linear servo system formed by dual parallel linear motors to drive the vertical axis ( $z$-axis) of a die-sinking electric discharge machine (EDM) tool. In the technique, apart from position control, the thrust outputs of the individual motors are also controlled equally to produce the overall thrust required so that one motor does not undergo a heavier load than the other for protection from potential overload. Also, the potential "pull-anddrag" effect causing the motors to oppose each other can be reduced.

The $z$-axis drive of the investigated EDM tool is shown in Fig. 1, where a pneumatic cylinder is used to actively compensate for the gravity and accelerative force of the two motor slides, the coupling and the quill (including the electrode and holder). These components driven by the

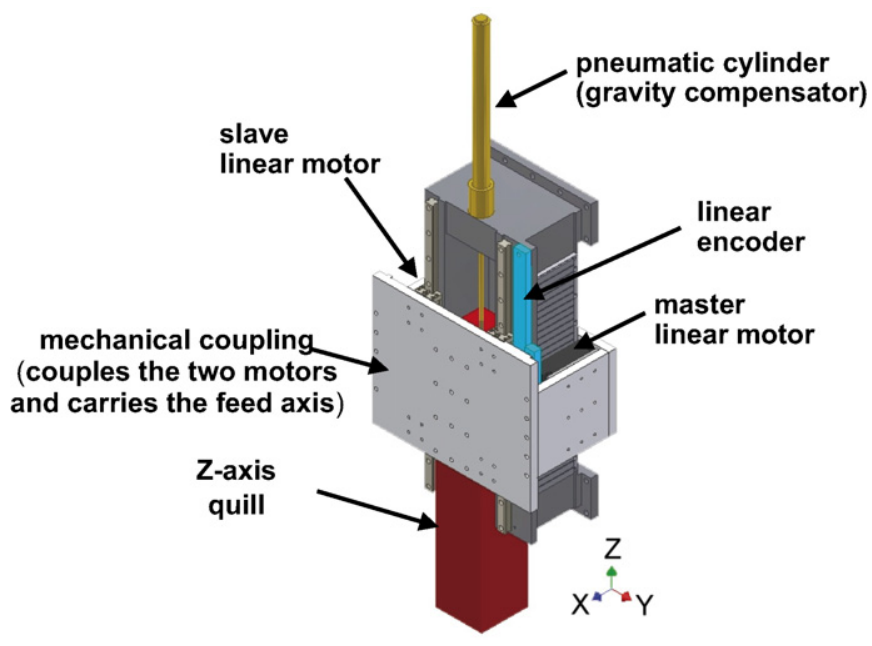

Fig. 1. Investigated EDM vertical axis. motors are hereafter called the "moving part". The overview of the experimental EDM in machining is shown in Fig. 2. The mechanical coupling shown in Fig. 1 strongly links the dual parallel linear motors so that only small nonsynchronization is allowed. For such a configuration, the master-slave control is often considered to be appropriate, where the slave motor is compliant to the master, and errors between them can be reduced by compensation. This compliance would effectively prevent the machine tool from potential hazards in comparison with the case where the stiffness control is applied to both motors. In the presented position/thrust hybrid synchronous control technique, the thrust compliance mode, instead of velocity, is used due to the consideration of thrust equality. The proposed control method aims to provide high speed, accuracy, and stiffness for the investigated EDM. Prior to the explanation of the control system and the presentation of its experimental results, it should be clarified that the term "synchronization error" is defined as the position differences between the dual parallel linear motors. In contrast, the term "tracking error," used in what follows, represents the error between the actual position and the desired position of the $z$-axis.

This paper is organized as follows: Section 2 introduces the pneumatic gravity compensator, followed by the design

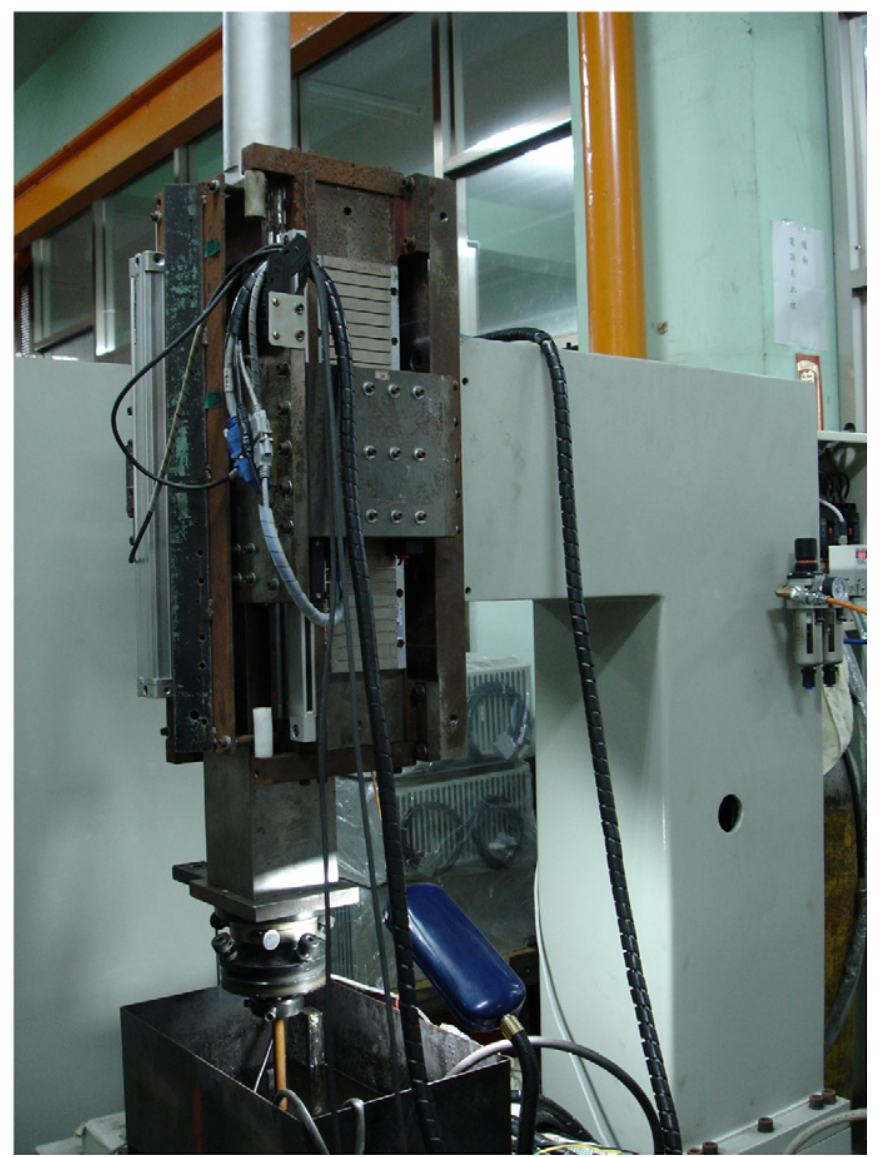

Fig. 2. Overview of the experimental EDM tool in machining. 
of the proposed control system in Section 3. The experimental study is discussed in Section 4 and, finally, conclusions are given at the end.

\section{Gravity compensator}

Pneumatic systems are widely used in industry to perform simple automation tasks due to the advantages of low cost, compact size, and safe actuation. For the investigated system presented in Figs. 1 and 2, the lowfriction pneumatic cylinder is employed to balance the gravitational and accelerative forces of the moving part (including the motor slides, the coupling and the $z$-axis quill with the electrode and holder). The pneumatic cylinder with an electro-pneumatic proportional valve is controlled by a built-in commercial controller, which is fed with current commands. Therefore, a simple voltagecurrent converter is built such that the controller accepts voltage command from a digital signal processor (DSP). The voltage command from the DSP is calculated according to the balancing force required for the dead weight of the moving part. The built-in controller is capable of regulating and generating the correct compensation force according to the variation of the accelerations plus the dead weight. The force-speed characteristic of the pneumatic unit is shown in Fig. 3.

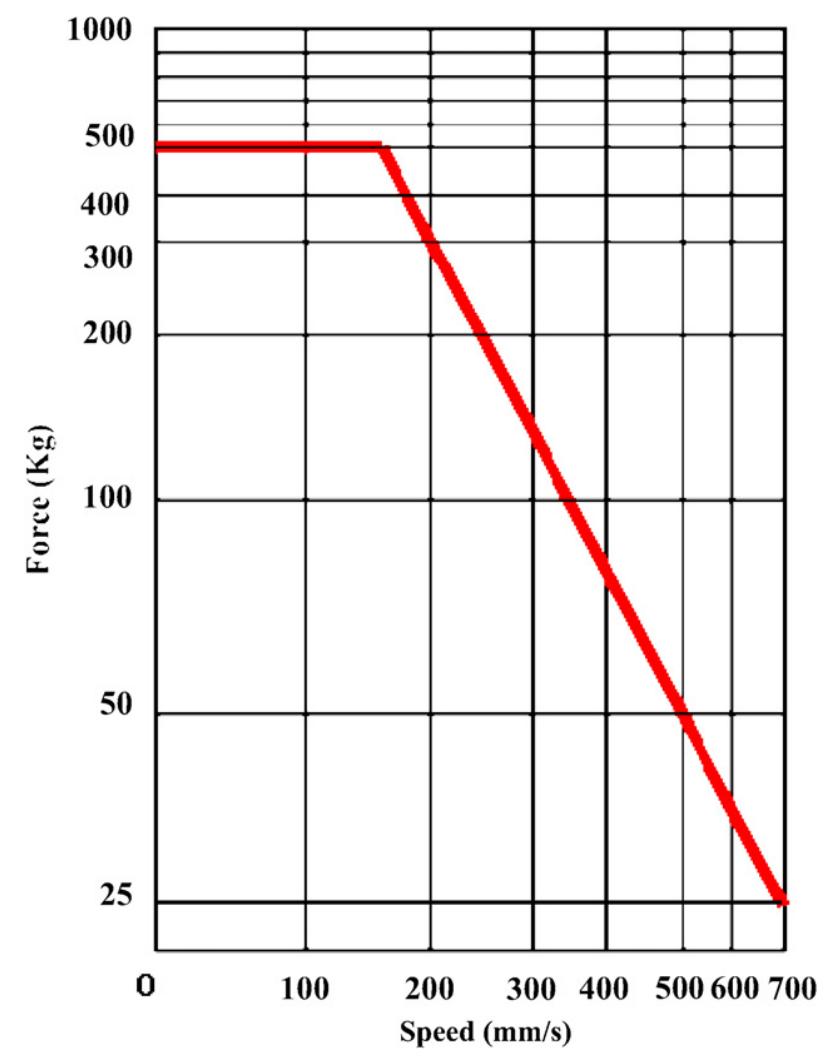

Fig. 3. Force-speed characteristic of the pneumatic cylinder.

\section{Control design}

As previously mentioned, both positioning accuracy and thrust balancing of the dual linear motors are considered in the proposed control design. The control diagram is shown in Fig. 4, where the master motor adopts high stiffness position control, and compliant thrust control is applied to the slave to follow desired motions represented as function of the master position. The mechanical coupling may be deformed due to the synchronization error between the two motors.

The deformed force is estimated based on the differences of the positions and estimated velocities between the two motors, and a compensator (i.e., the synchronous controller in Fig. 4) is designed to achieve a fast dynamic response without additional hardware. The control scheme is a MIMO system, which includes two input signals (the position command and gravity compensation command) and two output signals (the position of the master motor and the synchronization error of the two motors). The thrust command to the slave is calculated from the master motor whose thrust command is multiplied by a designed proportional gain before it is fed to the slave. This allows the slave motor which may have different characteristics from the master motor to output the thrust virtually equal to that of the master motor. The complete control design process is illustrated in the following.

\subsection{Identification of linear motors}

Prior to the design of the control parameters, the identification of the two motors is first conducted for servo loop design using the Control System Analyzer HP3563A. A swept sine voltage command generated by HP3563A is first given to drive the motor to be identified. This voltage command and the corresponding velocity voltage of the motor are then both fed back to HP3563A for analysis, and the transfer function can thus be determined via the curve-fitting technique. The identification results for the two motors are given as:

$G_{\mathrm{m}}=\frac{1702}{s+17.11}$,

$G_{\mathrm{s}}=\frac{1603}{s+12.07}$,

where $G_{\mathrm{m}}$ and $G_{\mathrm{s}}$ represent the transfer functions of the master and salve motors in the thrust mode, respectively. As can be seen, the two motors possess different characteristics and may respond differently to the same commands. This implies the need for the equal thrust control. Note that the identification is performed when the motors are disassembled from the EDM and hence excludes the effects of the coupling, the dead weight and the pneumatic cylinder. 


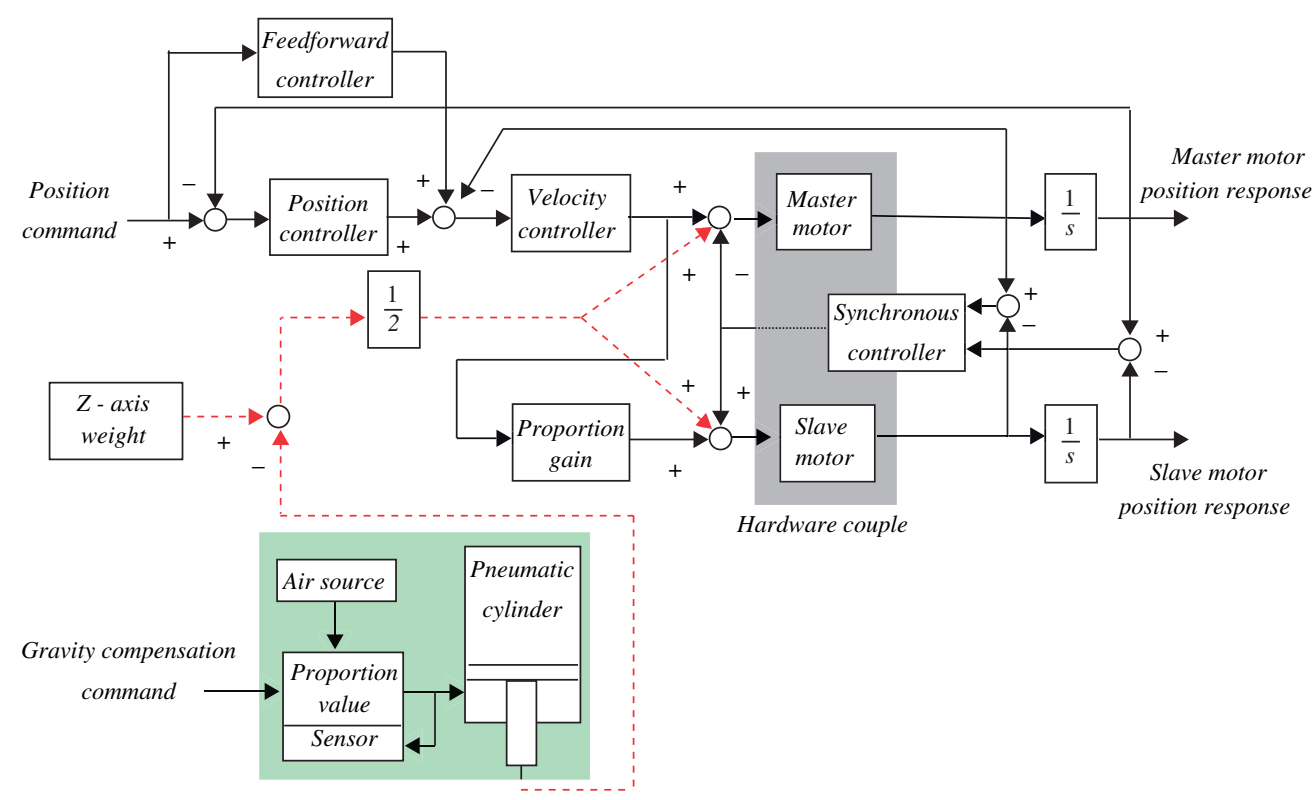

Fig. 4. Control diagram of the proposed position/thrust hybrid synchronous control.

\subsection{Control design for master motor}

The control design for the master motor should be conducted first to ensure the system positioning performance prior to design of any other controllers for the entire system shown in Fig. 4. The servo design techniques used in this paper are detailed in $[9,10]$. For the velocity control loop, a PI controller is used to achieve the required velocity response. The Pole-Zero cancellation technique [11] is applied to cancel the poles of the motor transfer function with the zeros of the PI controller so that the velocity openloop transfer function can be reduced to first order. The parameters of the designed PI controller can then be determined simply with the given velocity closed-loop bandwidth. The velocity controller is obtained as:

Velocity controller $=\frac{0.1 s+15.2}{s}$.

The position loop is designed based on the assumption that the bandwidth of the velocity loop is sufficiently greater than that of the position loop. In that case, the transfer function of the position loop becomes first-order and the control gain is equivalent to the bandwidth of the position loop. A velocity feedforward controller is added to improve the transient response and the system positioning tracking performance. The feedforward controller is designed to be $C_{\mathrm{f}}=s[9,10]$, which is then verified by a series of tests based on the presented control system. According to the $S$-curve experimental results presented in Fig. 5, $C_{\mathrm{f}}=0.98 \mathrm{~s}$ is capable of reducing the transient tracking error within $0.1 \mathrm{~mm}$ and the steady-state error within $1 \mu \mathrm{m}$, which is the resolution of the linear encoder. In comparison with the $C_{\mathrm{f}}=0.98 \mathrm{~s}$ case, the result for the designed $C_{\mathrm{f}}=s$ (not shown in Fig. 5) presents a smaller

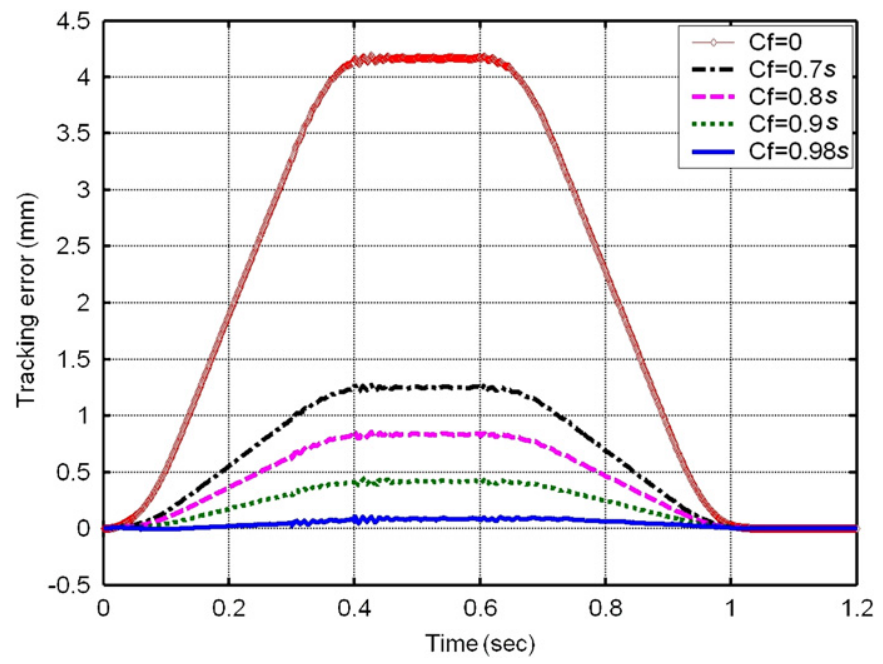

Fig. 5. Effect of the velocity feedforward controller.

tracking error of $-0.044-0.04 \mathrm{~mm}$, where the negative error is considered unacceptable. Consequently, $C_{\mathrm{f}}=0.98 \mathrm{~s}$ is adopted for further investigation.

The designed control parameters for the position loop and feedforward are listed as:

Position controller $=80$,

Feedforward controller $=0.98 s$.

\subsection{Thrust control design for slave motor}

The thrust control design for the slave motor is performed on completion of the design for the master. As shown in Fig. 4, a proportional gain is used to coordinate 
the thrust command of the two motors so that the overall required thrust can be equally shared by the two motors. Fig. 6 presents the relation between the proportional gain $K_{\mathrm{C}}$ and the two motor plants, where the input thrust commands and velocity output responses are denoted as $I_{\mathrm{m}}, I_{\mathrm{s}}, V_{\mathrm{m}}$, and $V_{\mathrm{s}}$, respectively. For the conditions of $I_{\mathrm{m}}=$ $I_{\mathrm{s}}$ and $G_{\mathrm{m}} \neq G_{\mathrm{s}}$, the output velocity $V_{\mathrm{m}}$ will not be equal to $V_{\mathrm{s}}$, as expected. Hence, for identical outputs produced

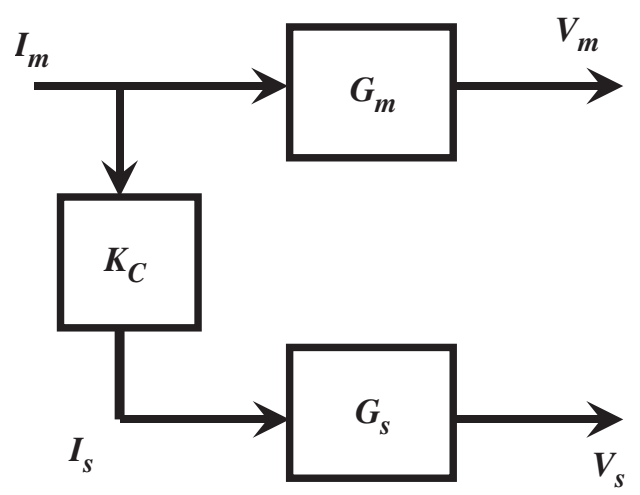

Fig. 6. Output relationship of the two motors.
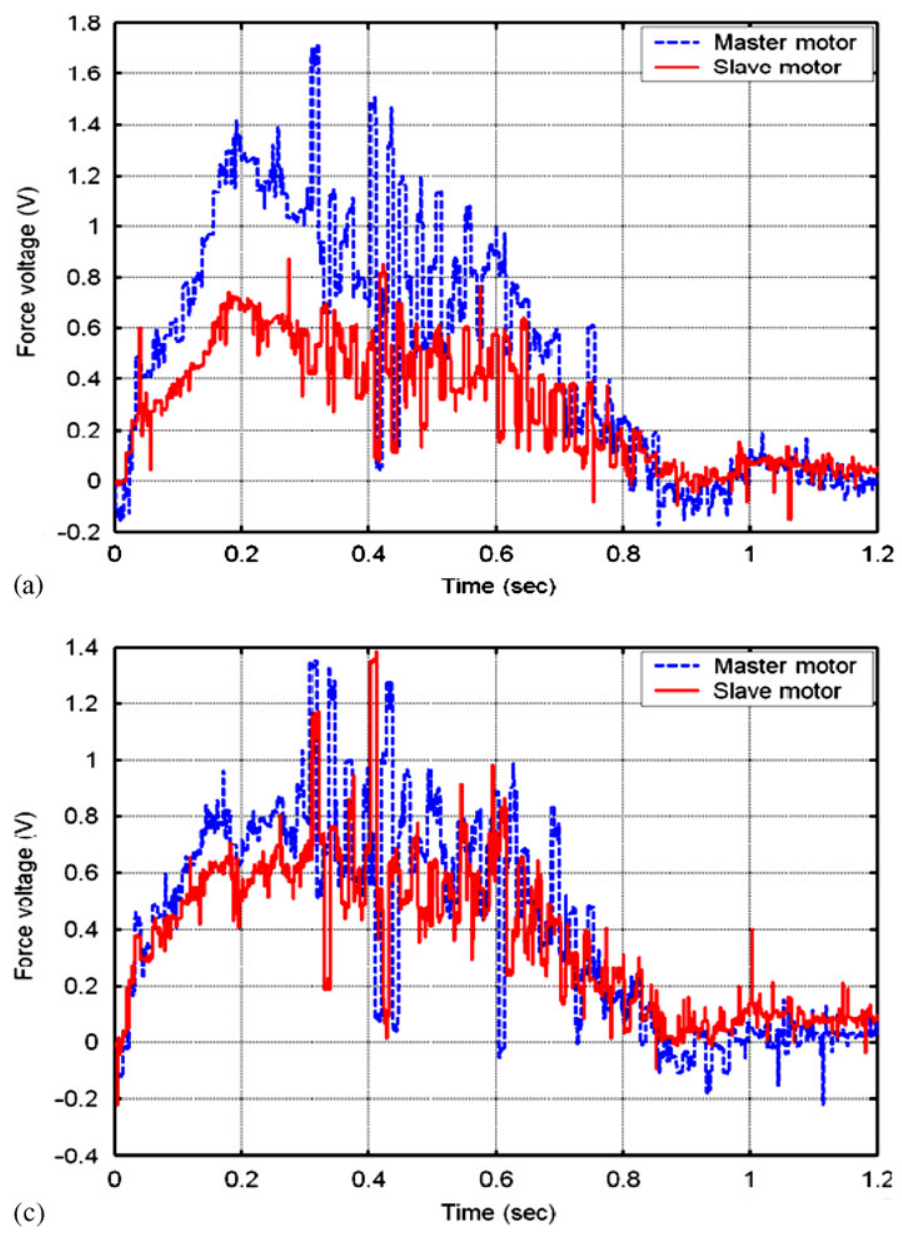

from the two motors, i.e., $V_{\mathrm{m}}=V_{\mathrm{s}}$, the following relationship is obtained.

$G_{\mathrm{m}} I_{\mathrm{m}}=G_{\mathrm{s}} I_{\mathrm{s}}$,

and

$I_{\mathrm{s}}=\frac{G_{\mathrm{m}}}{G_{\mathrm{s}}} I_{\mathrm{m}}=K_{\mathrm{C}} I_{\mathrm{m}}$

The two motors plants, $G_{\mathrm{m}}$ and $G_{\mathrm{s}}$, given in Eqs. (1) and (2) are rewritten by

$G_{\mathrm{m}}=\frac{b_{1}}{s+a_{1}}=\frac{1702}{s+17.11}$

and

$G_{\mathrm{s}}=\frac{b_{2}}{s+a_{2}}=\frac{1603}{s+12.07}$.

The proportional gain $K_{\mathrm{C}}$ is hence expressed by

$K_{\mathrm{C}}=\frac{G_{\mathrm{m}}}{G_{\mathrm{s}}}=\frac{b_{1} /\left(s+a_{1}\right)}{b_{2} /\left(s+a_{2}\right)}=\frac{b_{1}\left(s+a_{2}\right)}{b_{2}\left(s+a_{1}\right)}$.
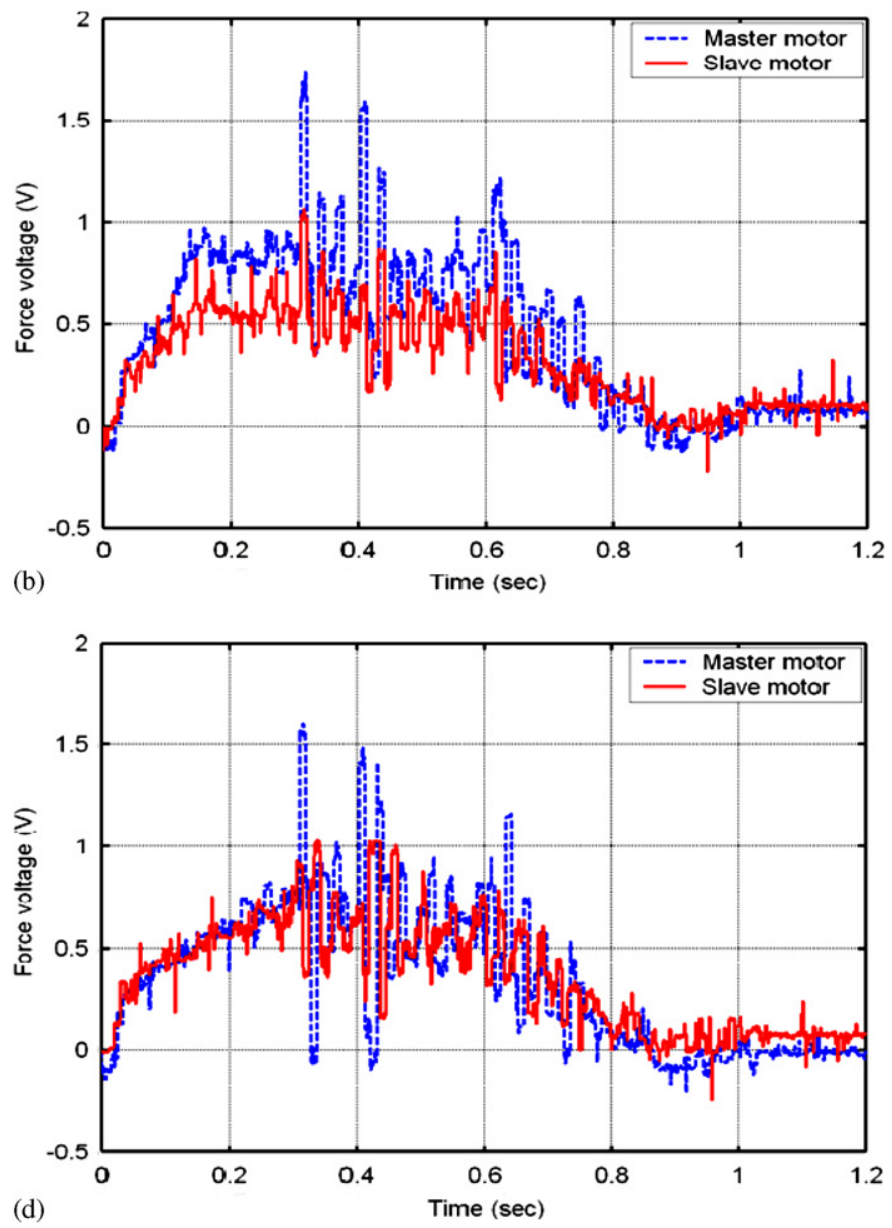

Fig. 7. Effect of $K_{\mathrm{C}}$ on thrust voltage outputs. (a) $K_{\mathrm{C}}=0.5$, (b) $K_{\mathrm{C}}=0.6$, (c) $K_{\mathrm{C}}=0.7$ and (d) $K_{\mathrm{C}}=0.8$. 
Finally, the static proportional gain $K_{\mathrm{C}}$ is determined to be:

$K_{\mathrm{C}}=\lim _{s \rightarrow 0} \frac{b_{1}\left(s+a_{2}\right)}{b_{2}\left(s+a_{1}\right)}=\frac{a_{2} b_{1}}{a_{1} b_{2}} \approx 0.75$.

The designed $K_{\mathrm{C}}=0.75$ indicates that the thrust command for the slave motor is unequal to that of the master. A series of tests with $K_{\mathrm{C}}$ varying from 0.5 to 1.0 has been conducted to study the effect of $K_{\mathrm{C}}$ on thrust sharing between the two motors. As the experimental results for $K_{\mathrm{C}}=0.5-0.8$ shown in Fig. 7 , the thrust outputs for the two motors appear to be more even when $K_{\mathrm{C}}=0.8$, which is close to the designed value 0.75 . The empirical value $K_{\mathrm{C}}=0.8$ is selected for further experiments on system performance.

\subsection{Design of synchronous controller}

The transient synchronization error between the master and slave motors is inevitable due to their inequality in characteristics and the environmental uncertainties. The situation can be even more severe when the two motors are coupled with a mechanism, which would cause the motors to oppose each other in a non-synchronization state. However, this can be improved by adding the synchronous controller (Fig. 4) to coordinate the movement of the motors [6]. Also, the strong mechanical coupling limits the relative movement of the two motors so that the synchronization error is expected to be small. Nevertheless, to avoid any potential hazard and strengthen the system safety, the synchronous controller is applied as an enhancement to the proposed compliant thrust control mode. The design of the synchronous controller is described in the following.

As seen in Fig. 8, the designed gains $\left(K_{1}\right.$ and $\left.K_{2}\right)$ are given for feeding the errors back to both the master and slave control loops. These two gains are treated as an equivalence to a PI controller $\left(\left(K_{2} s+K_{1}\right) / s\right)$ for design convenience. Using the Mason's gain formula, the transfer function of $I_{\mathrm{m}} \Rightarrow V_{\mathrm{m}}$ is obtained as

$\frac{V_{\mathrm{m}}}{I_{\mathrm{m}}}=\frac{A s^{2}+B s+C}{D s^{3}+E s^{2}+F s+G}$,

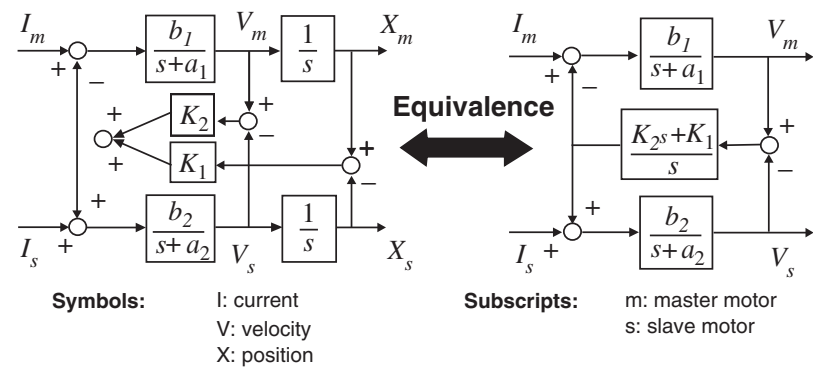

Fig. 8. Equivalent block diagrams for the two motors with coupling. where

$A=b_{1}$,

$B=a_{2} b_{1}+b_{1} b_{2} K_{2}$,

$C=b_{1} b_{2} K_{1}$,

$D=1$,

$E=a_{1}+a_{2}+b_{1} K_{2}+b_{2} K_{2}$,

$F=a_{1} a_{2}+b_{1} K_{1}+a_{2} b_{1} K_{2}+b_{2} K_{1}+a_{1} b_{2} K_{2}$,

$G=a_{2} b_{1} K_{1}+a_{1} b_{2} K_{1}$.

Therefore, $K_{1}=0.5$ and $K_{2}=0.05$ are determined with the known parameters $a_{1}, a_{2}, b_{1}$, and $b_{2}$ given in Eqs. (8) and (9). The model of the synchronous controller is shown in the following.

Synchronous controller $=0.5 \times($ synchronous position error $)$

$$
\begin{aligned}
& +0.05 \times \text { (synchronous velocity } \\
& \text { error). }
\end{aligned}
$$

The control system has been entirely constructed from the above discussion except for the pneumatic device which has a built-in controller.

\section{Experimental study}

The vertical feed axis of the investigated EDM shown in Fig. 1 has a $300 \mathrm{~mm}$ span across the two linear motors. The specification of the motors employed is shown in Table 1 . The axis formed by the two motors has a stroke of $200 \mathrm{~mm}$ and carries the moving part with a total weight of $120 \mathrm{Kg}$. Fig. 9 presents the system architecture of the experiment setup. The control system is implemented using the DSP control card, which also provides the control signal to the gravity compensator via a voltage-current converter as previously mentioned. For the experiments, three motion commands, two $S$-curve commands (low and high speeds) and a series of sinusoidal motion commands are given to evaluate the performance of the proposed control scheme. The low speed $S$ curve has a stroke of $200 \mathrm{~mm}$, a maximum speed of $200 / 0.6 \mathrm{~mm} / \mathrm{s}$, a maximum acceleration of $400 /$ $0.36 \mathrm{~mm} / \mathrm{s}^{2}$, and an average acceleration of $500 / 0.6 \mathrm{~mm} / \mathrm{s}^{2}$. With the same stroke, the high-speed command increases the maximum speed, maximum acceleration and average acceleration to $500 \mathrm{~mm} / \mathrm{s}, 2500 \mathrm{~mm} / \mathrm{s}^{2}$ and $500 / 0.3 \mathrm{~mm} / \mathrm{s}^{2}$, respectively. The technique for calculation of the $S$-curve

Table 1

Specification of the linear motors

Motor specification

Stroke length

Rated power

Maximum speed

Continuous stall force

Peak stall force
$0.2 \mathrm{~m}$

$1500 \mathrm{~W}$

$2 \mathrm{~m} / \mathrm{s}$

$600 \mathrm{~N}$

$1250 \mathrm{~N}$ 
Pneumatic cylinder
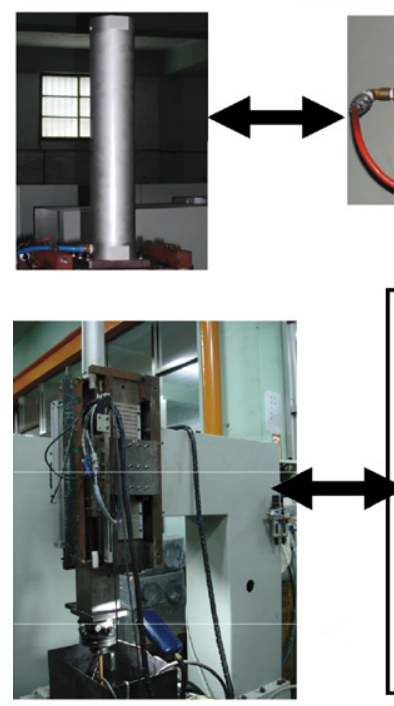

Experimental EDM
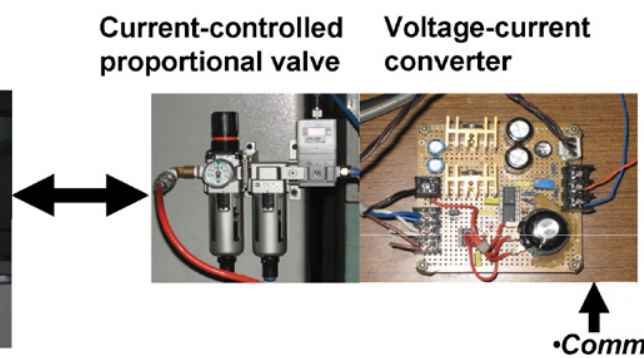

PC

Man-Machine interface

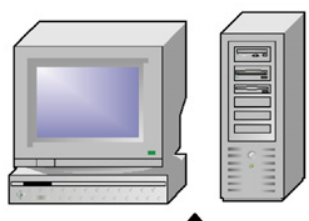

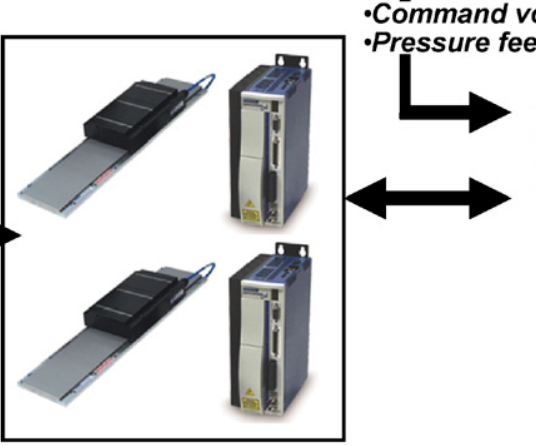

Linear motors and drivers

Fig. 9. System architecture of the experimental setup.

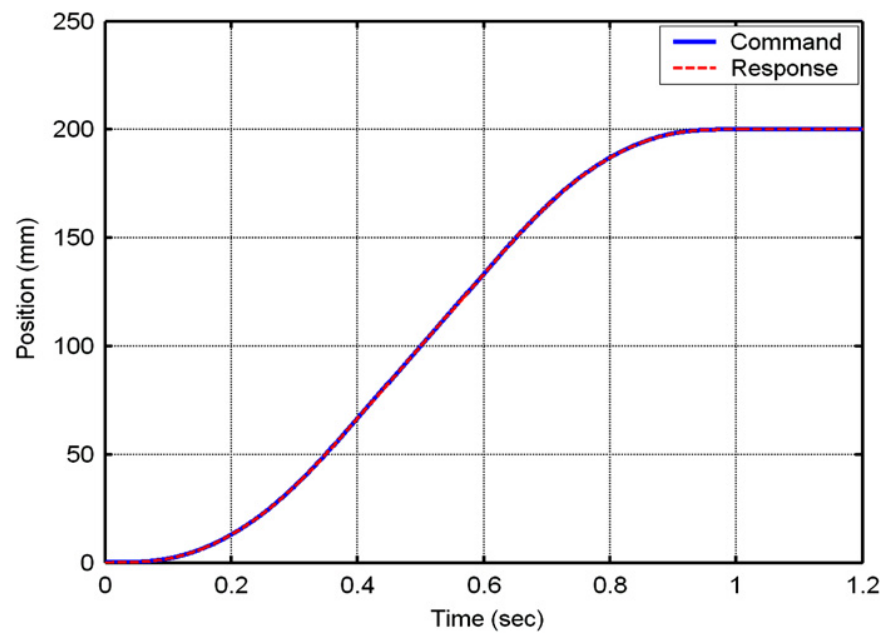

Fig. 10. Position response for low-speed $S$ curve command.

commands is given in Ref. [10]. The sine wave command has amplitude of $30 \mathrm{~mm}$ and frequencies varying from 1 to $4 \mathrm{~Hz}$. For fast removal of debris and increase of manufacturing speed, the $4 \mathrm{~Hz}, 30 \mathrm{~mm}$-amplitude sinusoidal motion is set as the targeted performance, which would require a theoretical peak acceleration of $1.93 \mathrm{~g}$ to be achieved.

The position commands and responses for the two planned $S$-curve motions are shown in Figs. 10 and 11, respectively. From Figs. 12 and 13, it is found that the transient tracking errors for the two cases are within 0.1 and $0.14 \mathrm{~mm}$, respectively, with both steady-state errors

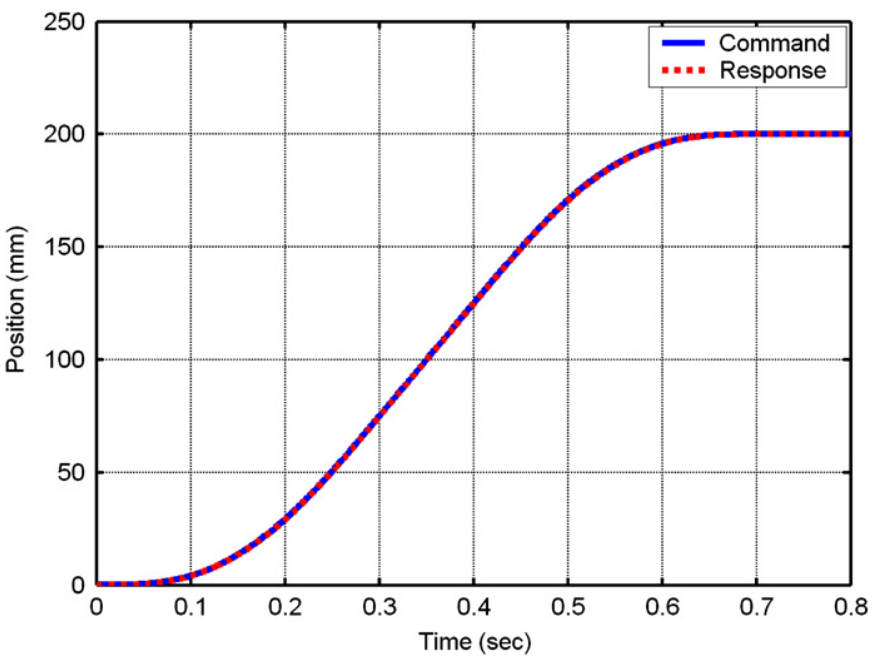

Fig. 11. Position response for high-speed $S$ curve command.

within $\pm 1 \mu \mathrm{m}$, which is the resolution of the linear encoder. This implies that, with the proposed control scheme, the positioning accuracy may possibly be further improved if an encoder with a higher resolution is used. Hence, the control system achieves a good performance in terms of the positioning accuracy.

The synchronization error for the two $S$-curve commands is controlled within a range from +5 to $-10 \mu \mathrm{m}$ approximately, as seen in Figs. 14 and 15, and that for the $4 \mathrm{~Hz}$ sinusoidal motion is +5 to $-3 \mu \mathrm{m}$, as shown in Fig. 16. The synchronization errors for the above three cases are considered significantly small in respect to the 


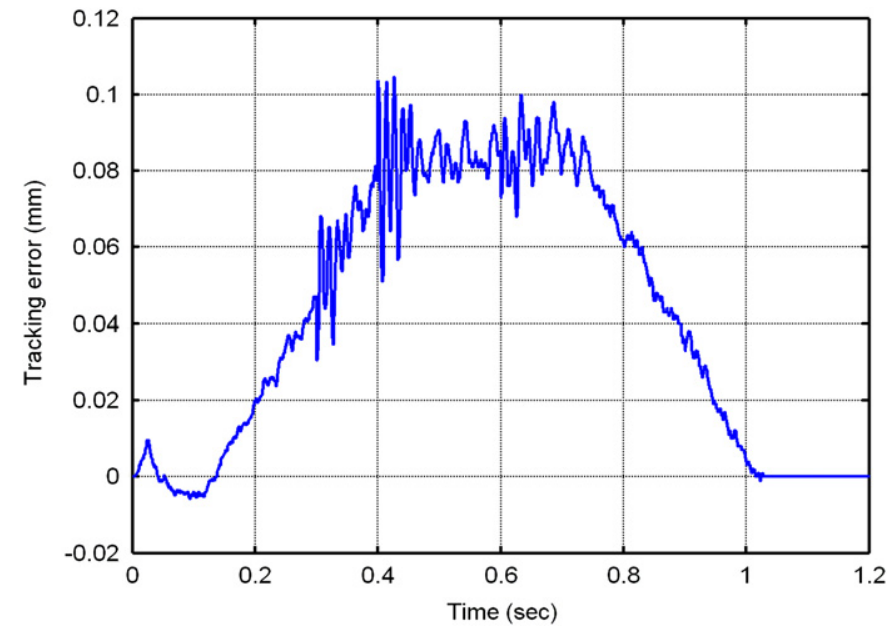

Fig. 12. Tracking error for low-speed $S$ curve command.

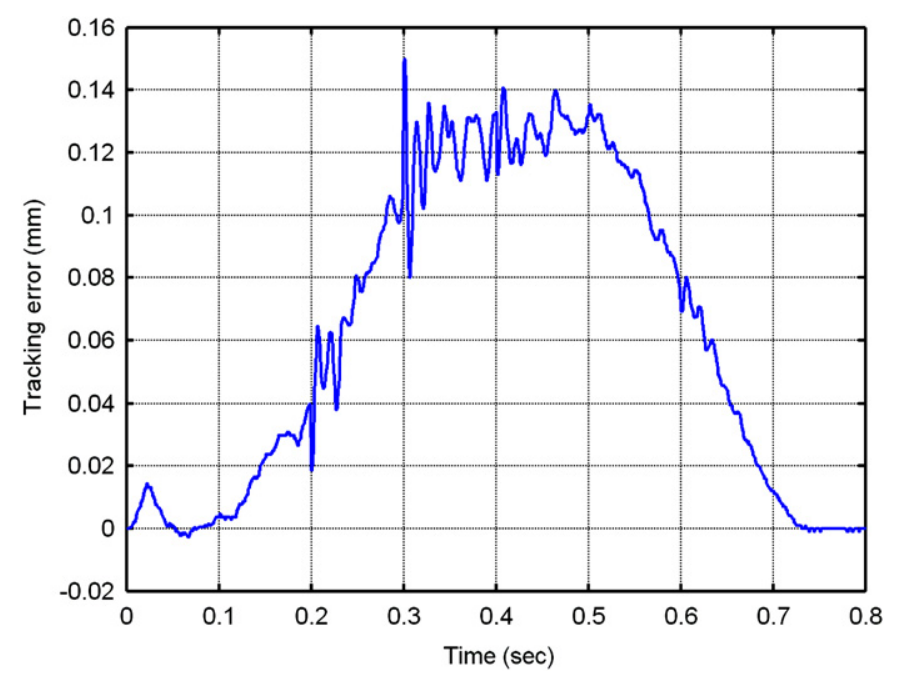

Fig. 13. Tracking error for high-speed $S$ curve command.

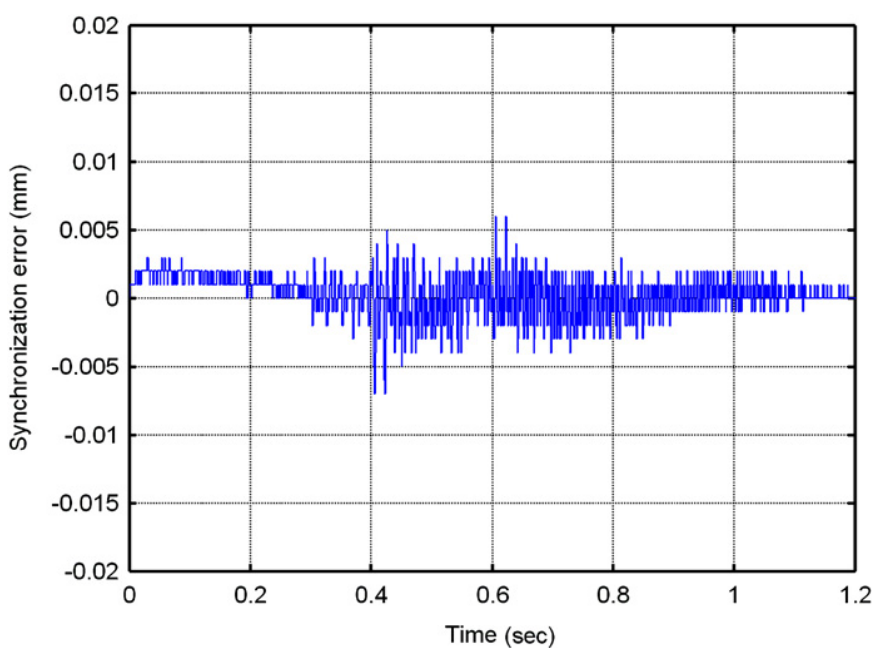

Fig. 14. Synchronization error for low-speed $S$ curve command.

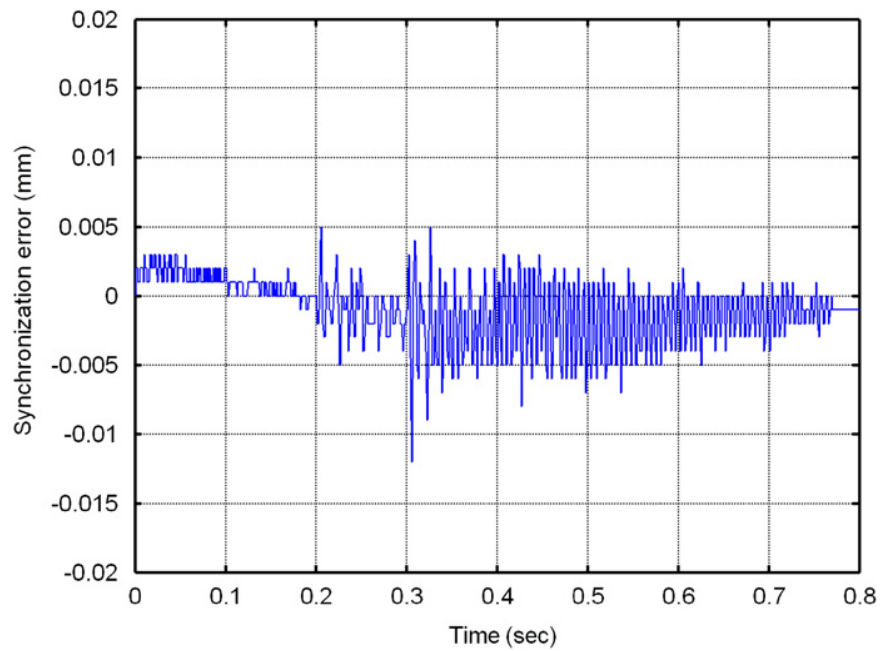

Fig. 15. Synchronization error for high speed $S$ curve command.

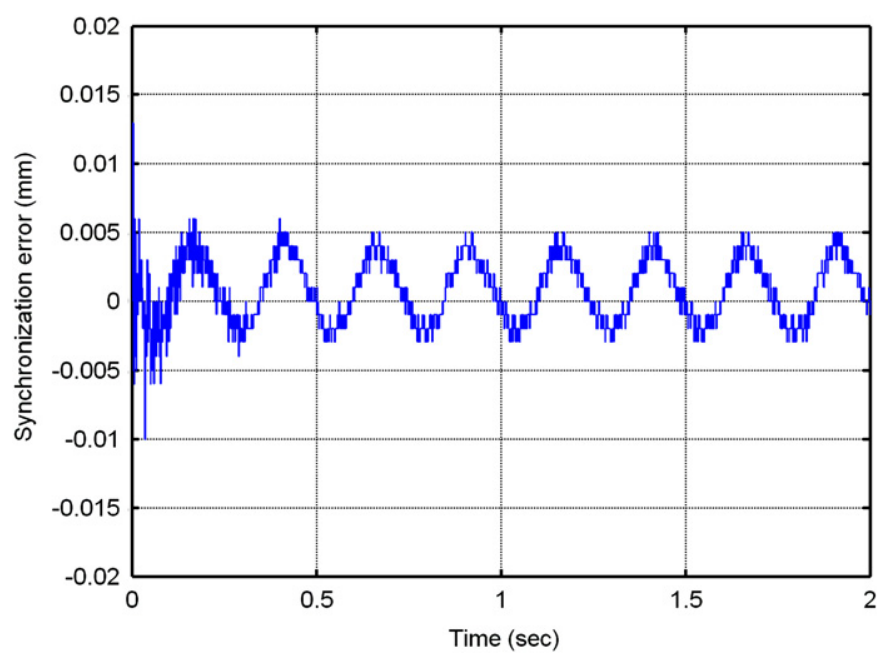

Fig. 16. Synchronization error for $4 \mathrm{~Hz}$ sine wave command.

$300 \mathrm{~mm}$ span between the two motors. Compared with the $4 \mathrm{~Hz}$ sinusoidal test, the results for the other sinusoidal cases have smaller magnitudes of errors and are not shown here.

Moreover, the experiment for the $4 \mathrm{~Hz}$ case shows a peak acceleration of $1.6 \mathrm{~g}$, which is lower than the theoretical acceleration of $1.93 \mathrm{~g}$ for that motion. This could be due to the effect of the pneumatic cylinder that possesses a slower response than the linear motors. However, the performance is considered satisfactory in comparison with few cases that can be found equipped with linear motors.

\section{Conclusion}

This paper has successfully constructed the servo system for the vertical axis of a die-sinking EDM tool using dual parallel linear motors with gravity compensation. An effective control scheme named the "position/thrust hybrid synchronous control" has been developed for the investigated system. The 
experimental results show that the synchronization errors for the planned motions are controlled within a range of +5 to $-10 \mu \mathrm{m}$. The steady-state position errors for the $S$-curve commands are also within the encoder resolution with a satisfactory tracking performance. For fast debris removal, the experiments also show that a satisfactory acceleration is achieved despite a potential effect of the slower pneumatic cylinder used. In addition, the purpose of achieving the desired thrust equality of the linear motors has also been illustrated and verified. Hence, it can be concluded that the proposed control scheme in this paper is capable of accomplishing high accuracy and high speed for the EDM investigated and could be applied to machine tools with a similar layout.

\section{Acknowledgment}

This work was supported in part by the National Science Council of Taiwan under Contract NSC 92-2213-E-006095. The EDM for experiments provided by Yawjet Enterprises Co., Ltd. is also acknowledged.

\section{References}

[1] R.B. Aronson, Attack of the linear motor, Manufacturing Engineering (1997) 60-71.
[2] M.C. Tsai, M.F. Hsieh, W.S. Yao, Synchronous control of linear servo systems for CNC machine tools, in: Proceedings of the 2003 European Control Conference, Cambridge, 2003.

[3] R.D. Lorenz, P.B. Schmidt, Synchronized motion control for process automation, in: Proceedings of the 1989 IEEE Industry Applications Annual Meeting, 1989, pp. 1693-1698.

[4] FANUC, Parameter Manual of $\alpha$-series AC Servo Motor, FANUC, Ltd., 1994.

[5] SIEMENS, 840D/FM-NC Description of Functions, Special Functions (Part 3), SIEMENS, 1999.

[6] J.X. Yang, M.C. Tsai, M.F. Hsieh, Identification and control of a linear servo system, The Fourth International Symposium on Linear Drives for Industry Applications, Birmingham, 2003, pp. 351-354.

[7] S. Cetin, A. Okada, Y. Uno, Electrode jump motion in linear motor equipped die-sinking EDM, Journal of Manufacturing Science and Engineering, Transactions of ASME 125 (2003) 809-815.

[8] Y. Zhao, X. Zhang, X. Lin, K. Yamazaki, Geometric modeling of the linear motor driven electrical discharge machining (EDM) die-sinking process, International Journal of Machine Tools Manufacture 44 (2004) 1-9.

[9] G. Ellis, Control System Design Guide, Academic Press, New York, 2000.

[10] H. Groß, J. Hamann, G. Wiegärtner, Electrical Feed Drives in Automation, Publicis MCD Corporate Publishing, Munich, 2001.

[11] K. Kim, R.C. Schaefer, Tuning a PID controller for a digital excitation control system, IEEE Transactions on Industry Applications 41 (2005) 485-492. 